Research Article

\title{
The Prevalence and Severity of Depression, Anxiety, and Stress among Medical Undergraduate Students of Arsi University and Their Association with Substance Use, Southeast Ethiopia
}

\author{
Leta Melaku $\mathbb{D}^{1},{ }^{1}$ Guta Bulcha, ${ }^{1}$ and Deresa Worku ${ }^{2}$ \\ ${ }^{1}$ Department of Biomedical Sciences, College of Health Sciences, Arsi University, Asella, Oromia, Ethiopia \\ ${ }^{2}$ Department of Clinical Nursing, College of Health Sciences, Arsi University, Asella, Oromia, Ethiopia \\ Correspondence should be addressed to Leta Melaku; letamelaku@gmail.com
}

Received 27 March 2021; Revised 12 July 2021; Accepted 19 July 2021; Published 27 July 2021

Academic Editor: Gwo-Jen Hwang

Copyright (c) 2021 Leta Melaku et al. This is an open access article distributed under the Creative Commons Attribution License, which permits unrestricted use, distribution, and reproduction in any medium, provided the original work is properly cited.

\begin{abstract}
Background. Mental problems can negatively impact physical and psychological well-being of junior medical students and predispose them to many unhealthy behaviors. Objective. We aimed to determine the prevalence and severity of depression, anxiety, and stress among medical undergraduate students of Arsi University and their association with substance use. Methods. Institutional-based cross-sectional study was conducted on 265 sampled medical students. Participants were selected by systematic random sampling. Data were collected by a pretested self-administrative questionnaire and analyzed using SPSS-21 software. Logistic regression analysis was employed, and statistical significance was accepted at $p<0.05$. Result. In the present study, 5 questionnaires were rejected for incompleteness giving response rate of $98.1 \%$. The mean age was $22.03(\mathrm{SD}=2.074)$ years. The current prevalence rates of depression, anxiety, and stress were $52.3 \%, 60.8 \%$, and $40.4 \%$, respectively. The overall prevalence of khat chewing, cigarette smoking, and alcohol drinking was found to be $21.5 \%, 15.4 \%$, and $33.8 \%$, respectively. Depression was significantly associated with monthly income $[\mathrm{AOR}=2.13]$, residency $[\mathrm{AOR}=13.10]$, and alcohol drinking $[\mathrm{AOR}=1.68]$. Anxiety was associated with gender $[\mathrm{AOR}=0.51]$, marital status $[\mathrm{AOR}=0.46]$, educational year $[\mathrm{AOR}=20.43]$, residency $[\mathrm{AOR}=58.72]$, and cigarette smoking $[\mathrm{AOR}=2.60]$. Stress was significantly associated with monthly income $[\mathrm{AOR}=2.21]$, educational year $[\mathrm{AOR}=3.05]$, residency $[\mathrm{AOR}=4.82]$, khat chewing $[\mathrm{AOR}=1.90]$, and drinking alcohol $[\mathrm{AOR}=1.84]$. Conclusion. To sum up, depression, anxiety, and stress are common problems among medical students of Arsi University. Monthly income, residency, and alcohol drinking were identified as risk factors of both depression and stress. In addition to other mentioned factors, educational year and khat chewing were identified as risk factors of stress. However, gender, marital status, educational year, residency, and cigarette smoking were identified as risk factors of anxiety. Counselling and awareness creation are recommended.
\end{abstract}

\section{Introduction}

Stress is the generalized, nonspecific response of the body to any factor that overwhelms, or threatens to overwhelm, the body's compensatory abilities to maintain homeostasis. Stress that enhances physical or mental function is considered as eustress. Conversely, distress is persistent stress that results in cognitive, behavioral, and emotional disturbances like anxiety and depression [1]. Clinically, anxiety is characterized by intense feeling of dread, accompanied by somatic symptoms that indicate hyperactive autonomic nervous system, whereas depression manifests as loss of interest or pleasure, sadness, feelings of guilt or low selfworth, disturbed sleep or appetite, extreme tiredness, and poor concentration [2].

In fact, transition from childhood to young adulthood is often marked by beginning of studying in university where certain factors contribute to the development or perpetuation of high incidence of mental illness in undergraduate programs. It is commonly observed that undergraduate medical students and resident doctors experience high incidences of mental illness across the globe, for instance, the prevalence of stress within undergraduate medical students of the United States (26\%) [3], Malaysia (41.9\%) [4], and 
Ethiopia (52.4\%) [5]. Furthermore, prevalence of anxiety was $65.5 \%$ in Greece [6], 69\% in Beirut [7], and 56\% in India [8]. Furthermore, across 26 US universities, $17.3 \%$ met the criteria for depression, 7.8\% generalized anxiety disorder, and $4.1 \%$ panic disorder, and a total of $6.3 \%$ reported suicidal ideation [9]. In Sweden, the prevalence of depressive symptoms was $12.9 \%$ and a total of $2.7 \%$ of students had made suicidal attempts [10]. In Zimbabwe, $64.5 \%$ of firstyear medical students were found to be at various levels of stress and/or depression with $12 \%$ at risk of suicidal tendencies [11].

The excessive amount of stress had led to grave consequences such as temptation to cheat on exams, poor academic performance, difficulties in solving interpersonal conflict, decreased attention, increased incidence of errors, negligence, sleep problems, and low self-esteem. Infrequent exercise, alcohol drinking, smoking, khat chewing, sleep disorders, and eating poorly are also identified to be associated with increased stress $[12,13]$. Chronic nicotine intake affects the brain reward system function which results in depression and anxiety symptoms. Khat use was also further associated with more mood disturbances than experienced by those who did not use khat and significantly associated with depression, anxiety, and cortisol stress response [14]. Heavy alcohol consumption is also associated with anxiety disorder [15]. Retrieving knowledge about presence of stress, depression, and anxiety is therefore important in itself and, if found, should be given attention for timely intervention. However, very little is known about depression, anxiety, and stress among medical undergraduate students in Ethiopia. Therefore, this study aimed to determine the prevalence and severities of depression, anxiety, and stress among medical undergraduate students of Arsi University and their association with substance use.

\section{Materials and Methods}

Institutional-based cross-sectional design was conducted in Arsi University from January 03 to 31, 2019, among 265 sampled undergraduate medical students. Of the 265 sampled students learning in Arsi University, 260 (98.1\%) agreed to take part. The sample size was first estimated by single population proportion, thereafter through a correction formula since overall numbers of medical students in study area were $<10,000$. After proportional allocation of the calculated sample size to each academic year level, stratified random sampling was employed. The required respondents were chosen from each stratum by randomly selecting the first respondent based on their ID number and then every 4 intervals through systematic random sampling. All medical students in Arsi University were eligible to participate with exception to severely ill students and those who were out of town during the time of data collection. Ethical support letter was obtained from Arsi University ethical board. Informed consent was secured and participation was totally voluntary. The confidentiality was kept anonymous.

Data were collected by a self-administered questionnaire that comprises the following parts: (i) Sociodemographic profile includes age, gender, enrollment year, marital status, residence, income, religion, and ethnicity.

(ii) Depression, Anxiety, Stress Scale-21 (DASS-21) is a self-report tool designed to measure the emotional states of depression, anxiety, and stress over the last week [16]. Each of the three DASS scales contains 7 items (Table 1). The depression scale assesses dysphoria, hopelessness, devaluation of life, self-deprecation, lack of interest or involvement, anhedonia, and inertia; the anxiety scale assesses autonomic arousal, skeletal muscle effects, situational anxiety, and subjective experience of anxious effect, while the stress scale is sensitive to levels of chronic nonspecific arousal. It also measures difficulty relaxing, nervous arousal, and being easily agitated, irritable, and impatient. When completing the DASS-21, the respondent is required to indicate the presence of a symptom over the previous week. Each item is scored from 0 (did not apply to me) to 3 (applied to me very much or most of the time). Sum scores are computed by adding up the scores on the items per (sub)scale and multiplying them by a factor 2. Sum scores for the total DASS-total scale thus range between 0 and 120 , and those for each of the subscales may range between 0 and 42 . For the depression domain, normal scores range from 0 to 9 , while higher scores range from 10 to 28 . For the anxiety domain, the normal scores range between 0 and 7 , while pathological scores range from 8 to 20 . For the stress domain, the normal scores range from 0 to 14 , while the pathological scores range from 15 to 34. Cut-off scores of 60 and 21 are used for the total DASS score and for the depression subscale, respectively. Scores $\geq 60$ (for DASS-total) and $\geq 21$ (for the depression subscale) are labeled as "high" or "severe." These cut-off scores are derived from a set of severity ratings, proposed by Lovibond and Lovibond [17]. However, to determine the prevalence of DAS, DASS-21 scores with normal levels of depression, anxiety, and stress were coded as " 0 " (No) whereas those with mild, moderate, severe, or extremely severe levels were coded as " 1 " (Yes). These cut-off scores are derived from a set of severity ratings, proposed by Yeshaw and Mossie [18]. Various studies demonstrated that the DASS-21 was found to have strong internal consistency. Anxiety Cronbach's $\alpha$ was 0.80 ; depression was 0.80 ; stress was 0.77 ; overall was 0.88 [19]. Cronbach's $\alpha$ for the combination of the anxiety and depression was 0.57 , 0.70 for anxiety and stress, and 0.60 for depression and stress [20].

(iii) Drug Abuse Surveillance Test (DAST) is a 10-item self-administered tool that provides a quick assessment of drug use problems [21]. In the present study, khat chewer is the proportion of individuals who had ever used khat at least once in his/her lifetime. Cigarette smoker is the proportion of 
TABLE 1: Items of depression, anxiety, stress scale-21 with their respective scores.

\begin{tabular}{lccc}
\hline & Depression & Anxiety & Stress \\
\hline Normal & $0-9$ & $0-7$ & $0-14$ \\
Mild & $10-13$ & $8-9$ & $15-18$ \\
Moderate & $14-20$ & $10-14$ & $19-25$ \\
Severe & $21-27$ & $15-19$ & $26-33$ \\
Extremely severe & $28+$ & $20+$ & 34 \\
\hline
\end{tabular}

individuals who had ever used cigarette at least once in his/her lifetime. Alcohol drinker is the proportion of individuals who had ever used alcohol drinks such as tela, tej, katicala/areke, beer, wine, or other drinks that can cause intoxication at least once in his/her lifetime.

2.1. Data Processing, Analysis, and Interpretation. The questionnaire was pretested on 13 randomly selected undergraduate medical students of Hawasa University. Data were checked for completeness on daily basis. To be edited and cleaned, the collected data were double entered into EpiData version 3.1 and exported into SPSS version 21 for analysis. Incomplete and inconsistent data were excluded from the analysis. The data was processed by using descriptive analysis and analytical methods, including frequency distribution, cross tabulation, and summary measures. Bivariate logistic regression was used to measure the association between independent variables and dependent variables. All variables with a $p$-value of $<0.05$ at bivariate logistic regression were entered into the multiple logistic regressions model for predicting the influence on criterion variables. Multiple logistic regressions analysis was carried out to find the role of each significant variable in determining the relevant subscale scores. Odds ratios were calculated and statistical significance was accepted at $p<0.05$.

\section{Results}

3.1. Sociodemographic Characteristics. From a total of 265 medical students who received the questionnaire, 260 completed the survey, yielding an overall response rate of $98.1 \%$. Age of the study sample ranged between 18 and 27 years with the mean (SD) of $22.03( \pm 2.62)$ years. In the present study, most respondents were male $(63.1 \%)$, single in marital status (75.4\%), living in campus (91.5\%), and having monthly income of $\leq 700$ ETB (52.7\%) (Table 2). Regarding their academic year, religion, and ethnicity, 54 (20.8\%) were from the sixth year, 130 (50\%) were Orthodox believers, and 139 (53.9\%) were Oromo, respectively.

3.2. Prevalence of Depression, Anxiety, and Stress. The present study demonstrated a strong internal consistency between depression and anxiety $(r=0.494, p<0.001)$, depression and stress $(r=0.456, p<0.001)$, and stress and anxiety $(r=0.420, p<0.001)$. From 260 valid analyzed questionnaires, the proportions of respondents detected to have depression, anxiety, and stress symptoms were $52.3 \%$, $60.8 \%$, and $40.4 \%$, respectively, whereas the percentages of respondents who had extremely severe symptoms of depression, anxiety, and stress were $6.2 \%, 16.2 \%$, and $2.3 \%$, respectively (Table 3 ).

3.3. Prevalence of Substance Use. In the present study, the overall prevalence of khat chewing, cigarette smoking, and alcohol drinking was found to be $21.5 \%, 15.4 \%$, and $33.8 \%$, respectively. Moreover, $32.1 \%$ of respondents chewed khat for 2-4 years, $35.0 \%$ smoked cigarette for 1-12 months, and $55.7 \%$ drank alcohol for $>4$ years. Finally, $44.6 \%$ of khat chewers and $75 \%$ of alcohol drinkers were occasional users while $32.5 \%$ of cigarette smoker smoked cigarette once a week (Table 4).

3.4. Association of Sociodemographic Characteristics and Use of Substance with Depression. A binary logistic regression analysis was applied to evaluate the possible impact of sociodemographic characteristics and behavioral factors on respondents' depression level (Table 5). In the present study, depression had no statistical association with age, educational year, religion, and ethnicity. Conversely, it had a statistically significant association with respondents' gender, in which males were 0.59 times less likely to be depressed than females (95\% CI: 0.36-0.99). It was also associated with monthly income, in which respondents who had monthly income of $\leq 700 \mathrm{ETB}$ were 1.68 times more depressed than above 700 ETB (95\% CI: 1.03-2.74). Depression was also associated with marital status, in which respondents who were not in a relationship (single) were 0.53 times less likely to be depressed than those in a relationship (married) $(95 \%$ CI: 0.30-0.95). Finally, it was also statistically associated with residency, in which nondormitory living respondents were 10.52 more likely to be depressed than those living in dormitory (95\% CI: 2.41-46.00).

Concerning substance use/behavioral factors, depression had statistical association with khat chewing: respondents who chewed khat were 2.07 times more likely to be depressed than nonchewers (95\% CI: 1.11-3.83). It was also statistically associated with cigarette smoking: respondents who smoke cigarettes were 2.42 times more likely to be depressed than nonsmokers (95\% CI: 1.17-5.00). Lastly, it was also associated with alcohol drinking: participants who drink alcohol were 2.01 times more likely to be depressed than nondrinkers (95\% CI: 1.19-3.41).

3.5. Association of Sociodemographic Characteristics and Use of Substance with Anxiety. In a binary logistic regression analysis of the present study, anxiety had no significant statistical association with sociodemographic variables like monthly income, religion, and ethnicity (Table 6). Contrariwise, it was statistically associated with the age of respondents, with individuals in the age range between 20 and 24 being 1.92 times less likely to be anxious than respondents $>24$ years ( $95 \% \mathrm{Cl}: 0.34-0.99)$. It was also associated with the 
TABLE 2: Sociodemographic characteristics in relation to gender, $N=260$, AU, January 2019.

\begin{tabular}{|c|c|c|c|c|}
\hline \multicolumn{2}{|c|}{ Sociodemographic variables } & Male & Female & Total $(N=260)$ \\
\hline \multirow{3}{*}{ Age } & $<20$ years & $36(54.5 \%)$ & $30(45.5 \%)$ & $66(100.0 \%)$ \\
\hline & $20-24$ years & $91(67.4 \%)$ & $44(32.6 \%)$ & $135(100.0 \%)$ \\
\hline & $>24$ years & $37(62.7 \%)$ & $22(37.3 \%)$ & $59(100.0 \%)$ \\
\hline \multirow{2}{*}{ Marital status } & Single & $128(65.3 \%)$ & $68(34.7 \%)$ & $196(100.0 \%)$ \\
\hline & Married & $36(56.3 \%)$ & $28(43.8 \%)$ & $64(100.0 \%)$ \\
\hline \multirow{2}{*}{ Monthly income } & $\leq 700 \mathrm{ETB}$ & $87(63.5 \%)$ & $50(36.5 \%)$ & $137(100.0 \%)$ \\
\hline & $>700$ ETB & $77(62.6 \%)$ & $46(37.4 \%)$ & $123(100.0 \%)$ \\
\hline \multirow{6}{*}{ Educational year } & $1^{\text {st }}$ year & $27(61.4 \%)$ & $17(38.6 \%)$ & $44(100.0 \%)$ \\
\hline & $2^{\text {nd }}$ year & $22(48.9 \%)$ & $23(51.1 \%)$ & $45(100.0 \%)$ \\
\hline & $3^{\text {rd }}$ year & $23(59.0 \%)$ & $16(41.0 \%)$ & $39(100.0 \%)$ \\
\hline & $4^{\text {th }}$ year & $36(76.6 \%)$ & $11(23.4 \%)$ & $47(100.0 \%)$ \\
\hline & $5^{\text {th }}$ year & $23(74.2 \%)$ & $8(25.8 \%)$ & $31(100.0 \%)$ \\
\hline & $6^{\text {th }}$ year & $33(61.1 \%)$ & $21(38.9 \%)$ & $54(100.0 \%)$ \\
\hline \multirow{2}{*}{ Residency } & Nondormitory & $11(50.0 \%)$ & $11(50.0 \%)$ & $22(100.0 \%)$ \\
\hline & Dormitory & $153(64.3 \%)$ & $85(35.7 \%)$ & $238(100.0 \%)$ \\
\hline \multirow{4}{*}{ Religion } & Orthodox & $80(61.5 \%)$ & $50(38.5 \%)$ & $130(100.0 \%)$ \\
\hline & Muslim & $40(62.5 \%)$ & $24(37.5 \%)$ & $64(100.0 \%)$ \\
\hline & Protestant & $42(67.7 \%)$ & $20(32.3 \%)$ & $62(100.0 \%)$ \\
\hline & Others ${ }^{E}$ & $2(50.0 \%)$ & $2(50.0 \%)$ & $4(100.0 \%)$ \\
\hline \multirow{7}{*}{ Ethnicity } & Oromo & $97(69.8 \%)$ & $42(30.2 \%)$ & $139(100.0 \%)$ \\
\hline & Amhara & $38(53.5 \%)$ & $33(46.5 \%)$ & $71(100.0 \%)$ \\
\hline & Sidama & $2(33.3 \%)$ & $4(66.7 \%)$ & $6(100.0 \%)$ \\
\hline & Tigre & $7(50.0 \%)$ & $7(50.0 \%)$ & $14(100.0 \%)$ \\
\hline & Wolayita & $5(62.5 \%)$ & $3(37.5 \%)$ & $8(100.0 \%)$ \\
\hline & Gurage & $12(66.7 \%)$ & $6(33.3 \%)$ & $18(100.0 \%)$ \\
\hline & Others $^{\dagger \dagger}$ & $3(75.0 \%)$ & $1(25.0 \%)$ & $4(100.0 \%)$ \\
\hline
\end{tabular}

${ }^{E}$ Catholic, Waqefatta, faith, and pagan. ${ }^{\dagger \dagger}$ Silte, Harari, and Kambata. ETB = Ethiopian Birr. Current exchange rate: $\$ 1$ USD $=36.05$ ETB.

TABle 3: Severity distribution of depression, anxiety, and stress in relation to gender, $N=260$, AU, January 2019.

\begin{tabular}{|c|c|c|c|c|}
\hline & \multirow{2}{*}{ Subscale } & \multicolumn{2}{|c|}{ Sex } & \multirow{2}{*}{ Total } \\
\hline & & Male $N(\%)$ & Female $N(\%)$ & \\
\hline \multirow{5}{*}{ Depression } & Normal & $86(69.4)$ & $38(30.6)$ & $124(100.0)$ \\
\hline & Mild & $34(63.0)$ & $20(37.0)$ & $54(100.0)$ \\
\hline & Moderate & $25(55.6)$ & $20(44.4)$ & $45(100.0)$ \\
\hline & Severe & $15(71.4)$ & $6(28.6)$ & $21(100.0)$ \\
\hline & Extremely severe & $4(25.0)$ & $12(75.0)$ & $16(100.0)$ \\
\hline \multirow{5}{*}{ Anxiety } & Normal & $72(70.6)$ & $30(29.4)$ & $102(100.0)$ \\
\hline & Mild & $13(59.1)$ & $9(40.9)$ & $22(100.0)$ \\
\hline & Moderate & $41(65.1)$ & $22(34.9)$ & $63(100.0)$ \\
\hline & Severe & $20(64.5)$ & $11(35.5)$ & $31(100.0)$ \\
\hline & Extremely severe & $18(42.9)$ & $24(57.1)$ & $42(100.0)$ \\
\hline \multirow{5}{*}{ Stress } & Normal & $106(68.4)$ & $49(31.6)$ & $155(100.0)$ \\
\hline & Mild & $20(54.1)$ & $17(45.9)$ & $37(100.0)$ \\
\hline & Moderate & $29(70.7)$ & $12(29.3)$ & $41(100.0)$ \\
\hline & Severe & $8(38.1)$ & $13(61.9)$ & $21(100.0)$ \\
\hline & Extremely severe & $1(16.7)$ & $5(83.3)$ & $6(100.0)$ \\
\hline
\end{tabular}

gender of respondents: males were 0.58 less likely to be anxious as compared with females (95\% Cl: 0.34-0.99). Anxiety was also associated with marital status: respondents who were not in a relationship (single) were 0.52 times less likely to be anxious than those in a relationship (married) (95\% CI: 0.28-0.96). Concerning its association with the educational level of respondents, third-year respondents were 4.85 more likely to be anxious than sixth-year students (95\% CI: 1.93-12.19). Finally, anxiety was also associated with residency: nondormitory living respondents were 15.48 more likely to be anxious than those living in a dormitory (95\% CI: 2.05-117.00).

Concerning substance use/behavioral factors, anxiety had statistical association with khat chewing; respondents who chewed khat were 2.03 times more likely to be anxious than nonchewers (95\% CI: 1.06-3.91). It was also statistically associated with cigarette smoking; respondents that smoke cigarettes were 2.52 times more likely to be anxious than 
TABLE 4: Distribution of behavioral factors/substance use among study participants in relation to gender, $N=260$, AU, January 2019.

\begin{tabular}{|c|c|c|c|c|}
\hline \multicolumn{2}{|c|}{ Substance use } & Khat chewer $N(\%)$ & Cigarette smoker $N(\%)$ & Alcohol drinker $N(\%)$ \\
\hline \multirow{4}{*}{ Duration } & $<1$ month & $12(21.4)$ & $9(22.5)$ & $9(10.2)$ \\
\hline & $1-12$ months & $14(25)$ & $14(35.0)$ & $8(9.1)$ \\
\hline & $2-4$ years & $18(32.1)$ & $11(27.5)$ & $22(25)$ \\
\hline & $>4$ years & $12(21.4)$ & $6(15)$ & $49(55.7)$ \\
\hline \multirow{4}{*}{ Frequency } & Every day & $10(17.9)$ & $12(30)$ & $0(0)$ \\
\hline & 2-3 days per week & $13(23.2)$ & $5(12.5)$ & $6(6.8)$ \\
\hline & Once a week & $8(14.3)$ & $13(32.5)$ & $16(18.2)$ \\
\hline & Occasionally & $25(44.6)$ & $10(25)$ & $66(75)$ \\
\hline
\end{tabular}

TABle 5: Bivariate logistic regression of factors associated with depression, $N=260$, AU, January 2019.

\begin{tabular}{|c|c|c|c|c|c|}
\hline \multirow{2}{*}{\multicolumn{2}{|c|}{ Variables }} & \multirow{2}{*}{ Total N (\%) } & \multicolumn{2}{|c|}{ Depression } & \multirow{2}{*}{$\mathrm{COR}$} \\
\hline & & & Yes $N(\%)$ & No $N(\%)$ & \\
\hline \multirow{3}{*}{ Age } & $<20$ years & $66(100.0)$ & $35(53.0)$ & $31(47.0)$ & $1.09(0.54-2.20)$ \\
\hline & $20-24$ years & $135(100.0)$ & $71(52.6)$ & $64(47.4)$ & $1.07(0.58-1.98)$ \\
\hline & $>24$ years & $59(100.0)$ & $30(50.8)$ & $29(49.2)$ & $1.00^{*}$ \\
\hline \multirow{2}{*}{ Sex } & Male & $164(100.0)$ & $78(47.6)$ & $86(52.4)$ & $0.59(0.36-0.99)$ \\
\hline & Female & $96(100.0)$ & $58(60.4)$ & $38(39.6)$ & $1.00^{*}$ \\
\hline \multirow{2}{*}{ Monthly income } & $\leq 700 \mathrm{ETB}$ & $137(100.0)$ & $80(58.4)$ & $57(41.6)$ & $1.68(1.03-2.74)$ \\
\hline & $>700 \mathrm{ETB}$ & $123(100.0)$ & $56(45.5)$ & $67(54.5)$ & $1.00^{*}$ \\
\hline \multirow{2}{*}{ Marital status } & Single & $196(100.0)$ & $95(48.5)$ & $101(51.5)$ & $0.53(0.30-0.95)$ \\
\hline & Married & $64(100.0)$ & $41(64.1)$ & $23(35.9)$ & $1.00^{*}$ \\
\hline \multirow{6}{*}{ Educational year } & First year & $44(100.0)$ & $23(52.3)$ & $21(47.7)$ & $1.27(0.57-2.82)$ \\
\hline & Second year & $45(100.0)$ & $26(57.8)$ & $19(42.2)$ & $1.59(0.72-3.52)$ \\
\hline & Third year & $39(100.0)$ & $18(46.2)$ & $21(53.8)$ & $0.99(0.44-2.27)$ \\
\hline & Fourth year & $47(100.0)$ & $25(53.2)$ & $22(46.8)$ & $1.32(0.60-2.89)$ \\
\hline & Fifth year & $31(100.0)$ & $19(61.3)$ & $12(38.7)$ & $1.84(0.75-4.51)$ \\
\hline & Sixth year & $54(100.0)$ & $25(46.3)$ & $29(53.7)$ & $1.00^{*}$ \\
\hline \multirow{2}{*}{ Residency } & Nondormitory & $22(100.0)$ & $20(90.9)$ & $2(9.1)$ & $10.52(2.41-46.00)$ \\
\hline & Dormitory & $238(100.0)$ & $116(48.7)$ & $122(51.3)$ & $1.00^{*}$ \\
\hline \multirow{4}{*}{ Religion } & Orthodox & $130(100.0)$ & $68(52.3)$ & $62(47.7)$ & $4.39(0.43-44.51)$ \\
\hline & Muslim & $64(100.0)$ & $38(59.4)$ & $26(40.6)$ & $3.29(0.33-32.47)$ \\
\hline & Protestant & $62(100.0)$ & $29(46.8)$ & $33(53.2)$ & $2.64(0.26-26.76)$ \\
\hline & Others & $4(100.0)$ & $1(25.0)$ & $3(75.0)$ & $1.00^{*}$ \\
\hline \multirow{7}{*}{ Ethnicity } & Oromo & $139(100.0)$ & $67(48.2)$ & $72(51.8)$ & $0.93(0.13-6.79)$ \\
\hline & Amhara & $71(100.0)$ & $40(56.3)$ & $31(43.7)$ & $1.29(0.17-9.68)$ \\
\hline & Sidama & $6(100.0)$ & $3(50.0)$ & $3(50.0)$ & $1.00(0.08-12.56)$ \\
\hline & Tigre & $14(100.0)$ & $7(50.0)$ & $7(50.0)$ & $1.00(0.11-9.23)$ \\
\hline & Wolayita & $8(100.0)$ & $6(75.0)$ & $2(25.0)$ & $3.00(0.24-37.67)$ \\
\hline & Gurage & $18(100.0)$ & $11(61.1)$ & $7(38.9)$ & $1.57(0.18-13.86)$ \\
\hline & Others & $4(100.0)$ & $2(50.0)$ & $2(50.0)$ & $1.00^{*}$ \\
\hline \multirow{2}{*}{ Khat chewing } & Yes & $56(100.0)$ & $37(66.1)$ & $19(33.9)$ & $2.07(1.11-3.83)$ \\
\hline & No & $204(100.0)$ & $99(48.5)$ & $105(51.5)$ & $1.00^{*}$ \\
\hline \multirow{2}{*}{ Cigarette smoking } & Yes & $40(100.0)$ & $28(70.0)$ & $12(30.0)$ & $2.42(1.17-5.00)$ \\
\hline & No & $220(100.0)$ & $108(49.1)$ & $112(50.9)$ & $1.00^{*}$ \\
\hline \multirow{2}{*}{ Alcohol drinking } & Yes & $88(100.0)$ & $56(63.6)$ & $32(36.4)$ & $2.01(1.19-3.41)$ \\
\hline & No & $172(100.0)$ & $80(46.5)$ & $92(53.5)$ & $1.00^{*}$ \\
\hline
\end{tabular}

${ }^{*}$ Reference category.

nonsmokers (95\% CI: 1.15-5.55). Finally, anxiety was also associated with alcohol drinking; participants who drink alcohol were 1.75 times more anxious than nondrinkers (95\% CI: 1.01-3.01).

3.6. Association of Sociodemographic Characteristics and Use of Substance with Stress. In the present study, when the impact of sociodemographic characteristics on the stress level was evaluated by binary logistic regression analysis, stress had no association with religion and ethnicity (Table 7). But it had a significant statistical association with the age of respondents, with respondents younger than 20 years being 2.07 times more likely to be stressed than those older than 24 years (95\% CI: 1.01-4.27). It was also associated with sex of respondents with males being 0.57 times less likely to be stressed than females (95\% CI: 0.34-0.95). 
TABLe 6: Bivariate logistic regression of factors associated with anxiety, $N=260$, AU, January 2019.

\begin{tabular}{|c|c|c|c|c|c|}
\hline \multirow{2}{*}{\multicolumn{2}{|c|}{ Variables }} & \multirow{2}{*}{ Total $N=260$} & \multicolumn{2}{|c|}{ Anxiety } & \multirow{2}{*}{$\mathrm{COR}$} \\
\hline & & & Yes $N(\%)$ & No $N(\%)$ & \\
\hline \multirow{3}{*}{ Age } & $<20$ years & $66(100.0)$ & $41(62.1)$ & $25(37.9)$ & $1.70(0.83-3.46)$ \\
\hline & $20-24$ years & $135(100.0)$ & $88(65.2)$ & $47(34.8)$ & $1.94(1.04-3.61)$ \\
\hline & $>24$ years & $59(100.0)$ & $29(49.2)$ & $30(50.8)$ & $1.00^{*}$ \\
\hline \multirow{2}{*}{ Sex } & Male & $164(100.0)$ & $92(56.1)$ & $72(43.9)$ & $0.58(0.34-0.99)$ \\
\hline & Female & $96(100.0)$ & $66(68.8)$ & $30(31.3)$ & $1.00^{*}$ \\
\hline \multirow{2}{*}{ Monthly income } & $\leq 700 \mathrm{ETB}$ & $137(100.0)$ & $89(65.0)$ & $48(35.0)$ & $1.45(0.88-2.39)$ \\
\hline & $>700 \mathrm{ETB}$ & $123(100.0)$ & $69(56.1)$ & $54(43.9)$ & $1.00^{*}$ \\
\hline \multirow{2}{*}{ Marital status } & Single & $196(100.0)$ & $112(57.1)$ & $84(42.9)$ & $0.52(0.28-0.96)$ \\
\hline & Married & $64(100.0)$ & $46(71.9)$ & $18(28.1)$ & $1.00^{*}$ \\
\hline \multirow{6}{*}{ Educational year } & First year & $44(100.0)$ & $25(56.8)$ & $19(43.2)$ & $1.91(0.85-4.28)$ \\
\hline & Second year & $45(100.0)$ & $34(75.6)$ & $11(24.4)$ & $4.50(1.88-10.73)$ \\
\hline & Third year & $39(100.0)$ & $30(76.9)$ & $9(23.1)$ & $4.85(1.93-12.19)$ \\
\hline & Fourth year & $47(100.0)$ & $29(61.7)$ & $18(38.3)$ & $2.34(1.05-5.22)$ \\
\hline & Fifth year & $31(100.0)$ & $18(58.1)$ & $13(41.9)$ & $2.014(0.82-4.94)$ \\
\hline & Sixth year & $54(100.0)$ & $22(40.7)$ & $32(59.3)$ & $1.00^{*}$ \\
\hline \multirow{2}{*}{ Residency } & Nondormitory & $22(100.0)$ & $21(95.5)$ & $1(4.5)$ & $15.48(2.05-116.00)$ \\
\hline & Dormitory & $238(100.0)$ & $137(57.6)$ & $101(42.4)$ & $1.00^{*}$ \\
\hline \multirow{4}{*}{ Religion } & Orthodox & $130(100.0)$ & $75(57.7)$ & $55(42.3)$ & $0.93(0.40-2.17)$ \\
\hline & Muslim & $64(100.0)$ & $45(70.3)$ & $19(29.7)$ & $0.79(0.31-2.03)$ \\
\hline & Protestant & $62(100.0)$ & $34(54.8)$ & $28(45.2)$ & $0.77(0.31-1.90)$ \\
\hline & Others & $4(100.0)$ & $4(100.0)$ & $0(0.0)$ & $1.00^{*}$ \\
\hline \multirow{7}{*}{ Ethnicity } & Oromo & $139(100.0)$ & $82(59.0)$ & $57(41.0)$ & $1.44(0.20-10.51)$ \\
\hline & Amhara & $71(100.0)$ & $42(59.2)$ & $29(40.8)$ & $1.45(0.19-10.88)$ \\
\hline & Sidama & $6(100.0)$ & $5(83.3)$ & $1(16.7)$ & $5.00(0.27-91.52)$ \\
\hline & Tigre & $14(100.0)$ & $10(71.4)$ & $4(28.6)$ & $2.50(0.26-24.38)$ \\
\hline & Wolayita & $8(100.0)$ & $5(62.5)$ & $3(37.5)$ & $1.67(0.15-18.87)$ \\
\hline & Gurage & $18(100.0)$ & $12(66.7)$ & $6(33.3)$ & $2.00(0.22-17.89)$ \\
\hline & Others & $4(100.0)$ & $2(50.0)$ & $2(50.0)$ & $1.00^{*}$ \\
\hline \multirow{2}{*}{ Khat chewing } & Yes & $56(100.0)$ & $41(73.2)$ & $15(26.8)$ & $2.03(1.06-3.91)$ \\
\hline & No & $204(100.0)$ & $117(57.4)$ & $87(42.6)$ & $1.00^{*}$ \\
\hline \multirow{2}{*}{ Cigarette smoking } & Yes & $40(100.0)$ & $31(77.5)$ & $9(22.5)$ & $2.52(1.15-5.55)$ \\
\hline & No & $220(100.0)$ & $127(57.7)$ & $93(42.3)$ & $1.00^{*}$ \\
\hline \multirow{2}{*}{ Alcohol drinking } & Yes & $88(100.0)$ & $61(69.3)$ & $27(30.7)$ & $1.75(1.01-3.01)$ \\
\hline & No & $172(100.0)$ & $97(56.4)$ & $75(43.6)$ & $1.00^{*}$ \\
\hline
\end{tabular}

${ }^{*}$ Reference category.

Statistically, it was also associated with monthly income; participants who had monthly income of $\leq 700$ ETB were 1.87 times more likely to be stressed than those respondents who had monthly income of above 700 ETB (95\% CI: 1.13-3.10). Stress was also associated with marital status; respondents who were not in a relationship (single) were 0.55 times less likely to be stressed than those in a relationship (married) (95\%: 0.31-0.96). Concerning its association with the educational level of respondents, second-year respondents were 4.92 times more likely to be stressed than those in sixth year (95\% CI: 2.09-11.61). Finally, it is also statistically associated with residency; nondormitory living respondents were 3.52 times more likely to be stressed than those in dormitory (95\% CI: 1.38-8.97).

Concerning substance use/behavioral factors, stress had statistical association with khat chewing; respondents who chewed khat were 1.99 times more likely to be stressed than nonchewers (95\% CI: 1.09-3.61) (Table 7). Furthermore, it was statistically associated with cigarette smoking; respondents who smoke cigarettes were 2.02 times more likely to be stressed than nonsmokers (95\% CI: 1.02-3.98). Finally, stress was also associated with alcohol drinking; participants who drink alcohol were 2.10 times more likely to be stressed than nondrinkers (95\% CI: 1.24-3.54).

3.7. Predictor Risk Factors for Depression. From variables like gender, monthly income, marital status, residency, khat chewing, cigarette smoking, and alcohol drinking which were candidates for multiple logistic regressions ( $p$-value $<0.05)$, predictors of the depression were monthly income, residency, and alcohol drinking (Table 8). In our study, the odds of being depressed were markedly increased by 2.13 times among respondents who had monthly income of $\leq 700$ ETB compared to $>700$ ETB (95\% CI: $1.24-3.66)$. Similarly, the odds of being depressed were markedly increased by 13.10 times among respondents living in nondormitory compared to those living in dormitory (95\% CI: 2.82-60.70). Finally, the oddd of being depressed were 
TABLe 7: Bivariate logistic regression of factors associated with stress, $N=260$, AU, January 2019.

\begin{tabular}{|c|c|c|c|c|c|}
\hline \multirow{2}{*}{\multicolumn{2}{|c|}{ Variables }} & \multirow{2}{*}{ Total $N=260$} & \multicolumn{2}{|c|}{ Stress } & \multirow{2}{*}{ COR } \\
\hline & & & Yes $N(\%)$ & No $N(\%)$ & \\
\hline \multirow{3}{*}{ Age } & $<20$ years & $66(100.0)$ & $34(51.5)$ & $32(48.5)$ & $2.07(1.01-4.27)$ \\
\hline & $20-24$ years & $135(100.0)$ & $51(37.8)$ & $84(62.2)$ & $1.18(0.62-2.25)$ \\
\hline & $>24$ years & $59(100.0)$ & $20(33.9)$ & $39(66.1)$ & $1.00^{*}$ \\
\hline \multirow{2}{*}{ Sex } & Male & $164(100.0)$ & $58(35.4)$ & $106(64.6)$ & $0.57(0.34-0.95)$ \\
\hline & Female & $96(100.0)$ & $47(49.0)$ & $49(51.0)$ & $1.00^{*}$ \\
\hline \multirow{2}{*}{ Monthly income } & $\leq 700 \mathrm{ETB}$ & $137(100.0)$ & $65(47.4)$ & $72(52.6)$ & $1.87(1.13-3.10)$ \\
\hline & $>700$ ETB & $123(100.0)$ & $40(32.5)$ & $83(67.5)$ & $1.00^{*}$ \\
\hline \multirow{2}{*}{ Marital status } & Single & $196(100.0)$ & $72(36.7)$ & $124(63.3)$ & $0.55(0.31-0.96)$ \\
\hline & Married & $64(100.0)$ & $33(51.6)$ & $31(48.4)$ & $1.00^{*}$ \\
\hline \multirow{6}{*}{ Educational year } & First year & $44(100.0)$ & $15(34.1)$ & $29(65.9)$ & $1.03(0.45-2.40)$ \\
\hline & Second year & $45(100.0)$ & $32(71.1)$ & $13(28.9)$ & $4.92(2.09-11.61)$ \\
\hline & Third year & $39(100.0)$ & $10(25.6)$ & $29(74.4)$ & $0.69(0.28-1.72)$ \\
\hline & Fourth year & $47(100.0)$ & $18(38.3)$ & $29(61.7)$ & $1.24(0.55-2.81)$ \\
\hline & Fifth year & $31(100.0)$ & $12(38.7)$ & $19(61.3)$ & $1.26(0.50-3.16)$ \\
\hline & Sixth year & $54(100.0)$ & $18(33.3)$ & $36(66.7)$ & $1.00^{*}$ \\
\hline \multirow{2}{*}{ Residency } & Nondormitory & $22(100.0)$ & $15(68.2)$ & $7(31.8)$ & $3.52(1.38-8.97)$ \\
\hline & Dormitory & $238(100.0)$ & $90(37.8)$ & $148(62.2)$ & $1.00^{*}$ \\
\hline \multirow{4}{*}{ Religion } & Orthodox & $130(100.0)$ & $50(38.5)$ & $80(61.5)$ & $0.83(0.11-6.25)$ \\
\hline & Muslim & $64(100.0)$ & $29(45.3)$ & $35(54.7)$ & $0.63(0.09-4.58)$ \\
\hline & Protestant & $62(100.0)$ & $24(38.7)$ & $38(61.3)$ & $0.63(0.08-4.79)$ \\
\hline & Others & $4(100.0)$ & $2(50.0)$ & $2(50.0)$ & $1.00^{*}$ \\
\hline \multirow{7}{*}{ Ethnicity } & Oromo & $139(100.0)$ & $55(39.6)$ & $84(60.4)$ & $0.22(0.02-2.15)$ \\
\hline & Amhara & $71(100.0)$ & $28(39.4)$ & $43(60.6)$ & $0.22(0.02-2.19)$ \\
\hline & Sidama & $6(100.0)$ & $4(66.7)$ & $2(33.3)$ & $0.67(0.04-11.29)$ \\
\hline & Tigre & $14(100.0)$ & $6(42.9)$ & $8(57.1)$ & $0.25(0.02-3.04)$ \\
\hline & Wolayita & $8(100.0)$ & $2(25.0)$ & $6(75.0)$ & $0.11(0.01-1.78)$ \\
\hline & Gurage & $18(100.0)$ & $7(38.9)$ & $11(61.1)$ & $0.21(0.02-2.47)$ \\
\hline & Others & $4(100.0)$ & $3(75.0)$ & $1(25.0)$ & $1.00^{*}$ \\
\hline \multirow{2}{*}{ Khat chewing } & Yes & $56(100.0)$ & $30(53.6)$ & $26(46.4)$ & $1.99(1.09-3.61)$ \\
\hline & No & $204(100.0)$ & $75(36.8)$ & $129(63.2)$ & $1.00^{*}$ \\
\hline \multirow{2}{*}{ Cigarette smoking } & Yes & $40(100.0)$ & $22(55.0)$ & $18(45.0)$ & $2.02(1.02-3.98)$ \\
\hline & No & $220(100.0)$ & $83(37.7)$ & $137(62.3)$ & $1.00^{*}$ \\
\hline \multirow{2}{*}{ Alcohol drinking } & Yes & $88(100.0)$ & $46(52.3)$ & $42(47.7)$ & $2.10(1.24-3.54)$ \\
\hline & No & $172(100.0)$ & $59(34.3)$ & $113(65.7)$ & $1.00^{*}$ \\
\hline
\end{tabular}

${ }^{*}$ Reference category.

TABLE 8: Multiple logistic regressions of depression and predictor variables, $N=260$, AU, January 2019.

\begin{tabular}{|c|c|c|c|c|c|}
\hline \multirow{2}{*}{\multicolumn{2}{|c|}{ Predictor variables }} & \multirow{4}{*}{$\begin{array}{c}\text { Total N (\%) } \\
164(100.0) \\
96(100.0)\end{array}$} & \multicolumn{2}{|c|}{ Depression } & \multirow{2}{*}{$\mathrm{AOR}$} \\
\hline & & & Yes $N(\%)$ & No $N(\%)$ & \\
\hline \multirow{2}{*}{ Sex } & Male & & $78(47.6)$ & $86(52.4)$ & $0.57(0.33-0.99)$ \\
\hline & Female & & $58(60.4)$ & $38(39.6)$ & $1.00^{*}$ \\
\hline \multirow{2}{*}{ Monthly income } & $\leq 700 \mathrm{ETB}$ & $137(100.0)$ & $80(58.4)$ & $57(41.6)$ & $2.13(1.24-3.66)$ \\
\hline & $>700$ ETB & $123(100.0)$ & $56(45.5)$ & $67(54.5)$ & $1.00^{*}$ \\
\hline \multirow{2}{*}{ Marital status } & Single & $196(100.0)$ & $95(48.5)$ & $101(51.5)$ & $0.56(0.30-1.06)$ \\
\hline & Married & $64(100.0)$ & $41(64.1)$ & $23(35.9)$ & $1.00^{*}$ \\
\hline \multirow{2}{*}{ Residency } & Nondormitory & $22(100.0)$ & $20(90.9)$ & $2(9.1)$ & $13.10(2.82-60.70)$ \\
\hline & Dormitory & $238(100.0)$ & $116(48.7)$ & $122(51.3)$ & $1.00^{*}$ \\
\hline \multirow{2}{*}{ Khat chewing } & Yes & $56(100.0)$ & $37(66.1)$ & $19(33.9)$ & $1.33(0.54-3.28)$ \\
\hline & No & $204(100.0)$ & $99(48.5)$ & $105(51.5)$ & $1.00^{*}$ \\
\hline \multirow{2}{*}{ Cigarette smoking } & Yes & $40(100.0)$ & $28(70.0)$ & $12(30.0)$ & $2.05(0.75-5.60)$ \\
\hline & No & $220(100.0)$ & $108(49.1)$ & $112(50.9)$ & $1.00^{*}$ \\
\hline \multirow{2}{*}{ Alcohol drinking } & Yes & $88(100.0)$ & $56(63.6)$ & $32(36.4)$ & $1.68(1.00-3.08)$ \\
\hline & No & $172(100.0)$ & $80(46.5)$ & $92(53.5)$ & $1.00^{*}$ \\
\hline
\end{tabular}

${ }^{*}$ Reference category. 
markedly increased by 1.68 times among alcohol users compared to nonusers (95\% CI: $1.00-3.08$ ).

3.8. Predictor Risk Factors for Anxiety. Age, sex, marital status, educational year, residency, khat chewing, cigarette smoking, and alcohol drinking had $p<0.05$ that made them candidates for multiple logistic regressions. However, gender, marital status, educational year, residency, and cigarette smoking were found to be predictors of anxiety (Table 9). In the present study, the odds of being anxious were markedly decreased by 0.51 times among male compared to female participants (95\% CI: $0.27-0.94$ ). The odds of being anxious were also markedly decreased by 0.46 times among participants who were single compared to those who were married (95\% CI: 0.23-0.94). Moreover, the odds of being anxious were markedly decreased as educational level increased after the first year. For example, the odds of being anxious were markedly increased by 20.43 times in the second-year students compared to sixth-year students $(95 \%$ CI: 4.40-94.89). The odds of being anxious were also more likely increased by 58.72 times among participants living in nondormitory compared to those in dormitory (95\% CI: 6.33-544.87). Finally, the odds of being anxious were markedly increased by 2.60 times among cigarette smokers compared to nonsmokers (95\% CI: 1.01-8.41).

3.9. Predictor Risk Factors for Stress. In the present study, age, sex, monthly income, marital status, educational year, residency, khat chewing, drinking alcohol, and cigarette smoking were candidate for multiple logistic regressions ( $p$ value $<0.05$ ). However, monthly income, educational year, residency, khat chewing, and drinking alcohol were found to be predictors of stress (Table 10). The odds of being stressed were markedly increased by 2.21 times among participants who had monthly income of $\leq 700$ ETB compared to those who had above 700 ETB (95\% CI: 1.08-4.51). Similarly, the odds of being stressed were markedly increased by 3.05 times in second-year participants compared to sixth-year participants (95\% CI: 1.05-12.47). The odds of being stressed were also more likely increased by 4.82 times among respondents living in nondormitory compared to those in dormitory (95\% CI: 1.61-14.46). Also, the odds of being stressed were more likely increased by 1.90 times among khat chewers compared to nonchewers (95\% CI: 1.02-5.01). Finally, the odds of being stressed were more likely increased by 1.84 times among alcohol users compared to nonusers (95\% CI: $1.01-3.42)$.

\section{Discussion}

Severe mental health problems interfere with individuals' emotional and cognitive as well as social abilities that can lead to underemployment and reduced productivity. Depression, anxiety, and stress are now the major mental health problems that cause disability globally, and no one is immune to these problems. Therefore, the main aim of this study was to determine the prevalence and severity of depression, anxiety, and stress among medical undergraduate students of Arsi University and their association with substance use.

Medical students are overloaded with a tremendous amount of information with limited time for internalization-a new study environment with obligations to succeed especially during preclinical encounters. This greater degree of workload creates feeling of distress and disappointments that predispose students to have difficulties in solving problems, impaired judgments, absenteeism from class lesson, and breaking of their mental stability.

The overall prevalence of depression, anxiety, and stress symptoms was $52.3 \%, 60.8 \%$, and $40.4 \%$, respectively. The explanation for this could be that medical students are overburdened with knowledge and have little time to internalize it, as well as a new study environment with pressures to excel, particularly during preclinical interactions. This increasing workload generates dissatisfaction and disappointment, which leads to trouble dealing with problems, bad judgments, absenteeism from class, and a loss of mental stability in students. Actually, our present finding is almost similar to the prevalence reported by Basudan et al. [22], Kulsoom and Afsar [23], and Iqbal et al. [24]. Conversely, it is higher than study report of Faud et al. [25], Moutinho et al. [26], and Mehta et al. [27]. This difference could be due to difference in cultural perception of stressful factors, economic burden, very high tuition fees, lack of family support, and higher or lower "readiness" to report different complaint. In our present setup, academic counselling is not a common practice which may be also a contributor.

The proportions of respondents who had extremely severe symptoms of depression, anxiety, and stress were $6.2 \%, 16.2 \%$, and $2.3 \%$, respectively. This prevalence was similar to finding of Patil et al. [28]. However, it was inconsistent with study result of Iqbal et al. [24] and Al-Ani Radeef and Ghazi [29]. Discrepancies stemming from methodology and type of questionnaire used could account for this high prevalence obtained by the aforementioned authors. The other possible reasons for the variability could be due to certain differences in the curricula, teaching facilities, qualification and experience of the instructors, and levels of care given to the students.

In this cross-sectional study, we correlated sociodemographic risk factors of the student (as independent variables) with the prevalence of depression, anxiety, and stress level amongst the undergraduate medical students of Arsi University. In our study, students living in nondormitory have considerably higher degree of depression, anxiety, and stress, which infers that living in dormitory came out to be protective. This finding is in line with study reported by Rab et al. [30] and Shendarkar [31]. However, it is inconsistent with study report of Kunwar et al. [32] and Liaqat et al. [33]. This unequivocal distribution tells us that probably depression, anxiety, and stress are associated with a multitude of factors such as poor dormitory conditions, more economic stress, distance from the family, less structured environment, and problems dealing with roommates, which might be different or parallel in the two study groups under consideration. 
TABle 9: Multiple logistic regressions of anxiety and predictor variables, $N=260$, AU, January 2019.

\begin{tabular}{|c|c|c|c|c|c|}
\hline \multirow{2}{*}{\multicolumn{2}{|c|}{ Predictor variables }} & \multirow{2}{*}{ Total $N=260$} & \multicolumn{2}{|c|}{ Anxiety } & \multirow{2}{*}{$\mathrm{AOR}$} \\
\hline & & & Yes $N(\%)$ & No $N(\%)$ & \\
\hline \multirow{3}{*}{ Age } & $<20$ years & $66(100.0)$ & $41(62.1)$ & $25(37.9)$ & $0.31(0.07-1.46)$ \\
\hline & $20-24$ years & $135(100.0)$ & $88(65.2)$ & $47(34.8)$ & $0.56(0.21-1.54)$ \\
\hline & $>24$ years & $59(100.0)$ & $29(49.2)$ & $30(50.8)$ & $1.00^{*}$ \\
\hline \multirow{2}{*}{ Sex } & Male & $164(100.0)$ & $92(56.1)$ & $72(43.9)$ & $0.51(0.27-0.94)$ \\
\hline & Female & $96(100.0)$ & $66(68.8)$ & $30(31.3)$ & $1.00^{*}$ \\
\hline \multirow{2}{*}{ Marital status } & Single & $196(100.0)$ & $112(57.1)$ & $84(42.9)$ & $0.46(0.23-0.94)$ \\
\hline & Married & $64(100.0)$ & $46(71.9)$ & $18(28.1)$ & $1.00^{*}$ \\
\hline \multirow{6}{*}{ Educational year } & First year & $44(100.0)$ & $25(56.8)$ & $19(43.2)$ & $12.91(2.40-69.36)$ \\
\hline & Second year & $45(100.0)$ & $34(75.6)$ & $11(24.4)$ & $20.43(4.40-94.89)$ \\
\hline & Third year & $39(100.0)$ & $30(76.9)$ & $9(23.1)$ & $18.03(4.54-71.58)$ \\
\hline & Fourth year & $47(100.0)$ & $29(61.7)$ & $18(38.3)$ & $9.16(2.65-31.66)$ \\
\hline & Fifth year & $31(100.0)$ & $18(58.1)$ & $13(41.9)$ & $6.89(2.10-22.55)$ \\
\hline & Sixth year & $54(100.0)$ & $22(40.7)$ & $32(59.3)$ & $1.00^{*}$ \\
\hline \multirow{2}{*}{ Residency } & Nondormitory & $22(100.0)$ & $21(95.5)$ & $1(4.5)$ & $58.72(6.33-544.87)$ \\
\hline & Dormitory & $238(100.0)$ & $137(57.6)$ & $101(42.4)$ & $1.00^{*}$ \\
\hline \multirow{2}{*}{ Khat chewing } & Yes & $56(100.0)$ & $37(66.1)$ & $19(33.9)$ & $1.57(0.57-4.33)$ \\
\hline & No & $204(100.0)$ & $99(48.5)$ & $105(51.5)$ & $1.00^{*}$ \\
\hline \multirow{2}{*}{ Cigarette smoking } & Yes & $40(100.0)$ & $28(70.0)$ & $12(30.0)$ & $2.60(1.01-8.41)$ \\
\hline & No & $220(100.0)$ & $108(49.1)$ & $112(50.9)$ & $1.00^{*}$ \\
\hline \multirow{2}{*}{ Alcohol drinking } & Yes & $88(100.0)$ & $56(63.6)$ & $32(36.4)$ & $1.41(0.73-2.74)$ \\
\hline & No & $172(100.0)$ & $80(46.5)$ & $92(53.5)$ & $1.00^{*}$ \\
\hline
\end{tabular}

${ }^{*}$ Reference category.

TABLE 10: Multiple logistic regressions of stress and predictor variables, $N=260$, AU, January 2019.

\begin{tabular}{|c|c|c|c|c|c|}
\hline \multirow{2}{*}{\multicolumn{2}{|c|}{ Predictor variables }} & \multirow{2}{*}{ Total $N=260$} & \multicolumn{2}{|c|}{ Stress } & \multirow{2}{*}{ AOR } \\
\hline & & & Yes $N(\%)$ & No $N(\%)$ & \\
\hline \multirow{3}{*}{ Age } & $<20$ years & $66(100.0)$ & $34(51.5)$ & $32(48.5)$ & $1.94(0.42-8.92)$ \\
\hline & $20-24$ years & $135(100.0)$ & $51(37.8)$ & $84(62.2)$ & $0.90(0.35-2.33)$ \\
\hline & $>24$ years & $59(100.0)$ & $20(33.9)$ & $39(66.1)$ & $1.00^{*}$ \\
\hline \multirow{2}{*}{ Sex } & Male & $164(100.0)$ & $58(35.4)$ & $106(64.6)$ & $0.59(0.33-1.06)$ \\
\hline & Female & $96(100.0)$ & $47(49.0)$ & $49(51.0)$ & $1.00^{*}$ \\
\hline \multirow{2}{*}{ Monthly income } & $\leq 700 \mathrm{ETB}$ & $137(100.0)$ & $65(47.4)$ & $72(52.6)$ & $2.21(1.08-4.51)$ \\
\hline & $>700 \mathrm{ETB}$ & $123(100.0)$ & $40(32.5)$ & $83(67.5)$ & $1.00^{*}$ \\
\hline \multirow{2}{*}{ Marital status } & Single & $196(100.0)$ & $72(36.7)$ & $124(63.3)$ & $0.55(0.29-1.04)$ \\
\hline & Married & $64(100.0)$ & $33(51.6)$ & $31(48.4)$ & $1.00^{*}$ \\
\hline \multirow{6}{*}{ Educational year } & First year & $44(100.0)$ & $15(34.1)$ & $29(65.9)$ & $0.57(0.11-3.04)$ \\
\hline & Second year & $45(100.0)$ & $32(71.1)$ & $13(28.9)$ & $3.05(1.05-12.47)$ \\
\hline & Third year & $39(100.0)$ & $10(25.6)$ & $29(74.4)$ & $0.53(0.15-1.89)$ \\
\hline & Fourth year & $47(100.0)$ & $18(38.3)$ & $29(61.7)$ & $1.14(0.34-3.79)$ \\
\hline & Fifth year & $31(100.0)$ & $12(38.7)$ & $19(61.3)$ & $1.15(0.33-3.99)$ \\
\hline & Sixth year & $54(100.0)$ & $18(33.3)$ & $36(66.7)$ & $1.00^{*}$ \\
\hline \multirow{2}{*}{ Residency } & Nondormitory & $22(100.0)$ & $15(68.2)$ & $7(31.8)$ & $4.82(1.61-14.46)$ \\
\hline & Dormitory & $238(100.0)$ & $90(37.8)$ & $148(62.2)$ & $1.00^{*}$ \\
\hline \multirow{2}{*}{ Khat chewing } & Yes & $56(100.0)$ & $37(66.1)$ & $19(33.9)$ & $1.90(1.02-5.01)$ \\
\hline & No & $204(100.0)$ & $99(48.5)$ & $105(51.5)$ & $1.00^{*}$ \\
\hline \multirow{2}{*}{ Cigarette smoking } & Yes & $40(100.0)$ & $28(70.0)$ & $12(30.0)$ & $0.99(0.33-2.96)$ \\
\hline & No & $220(100.0)$ & $108(49.1)$ & $112(50.9)$ & $1.00^{*}$ \\
\hline \multirow{2}{*}{ Alcohol drinking } & Yes & $88(100.0)$ & $56(63.6)$ & $32(36.4)$ & $1.84(1.01-3.42)$ \\
\hline & No & $172(100.0)$ & $80(46.5)$ & $92(53.5)$ & $1.00^{*}$ \\
\hline
\end{tabular}

${ }^{*}$ Reference category.

Medical students who had lower monthly income were more likely to be depressed and stressed. Despite the fact that food and dormitory services are provided to the students by the university, students need money for excursions, to print handouts, and to buy dressings and other basic necessities. This indicates that financial constraints could be an 
additional source of depression, anxiety, and stress besides academic stressors. However, other studies have noted no difference $[34,35]$. Again, sample size differences may possibly account for these different results. The same stressors may be perceived differently by different medical students, depending on their cultural background, personal traits, experience, and coping skills. The educational system also plays an enabling role subsequently leading to increased stress levels experienced by students.

There was a significant association between students' academic year and level of anxiety and stress. Many studies have shown higher stress levels and a higher prevalence of stress-related illnesses among second-year medical students as compared with other academic years. On the other hand, senior students developed skills of how to manage stress and stress-related illness compared to students in the early years [36]. The possible reason could be the amount and complexity of the material to be learned in the second year with progressive assessments of anatomy, physiology, and biochemistry that they have to pass to join the next higher level. Additional supportive evidence is that high level of stress and stress-related illness can be attributed to course workload, lack of leisure time, shortage of learning materials, and frequent examinations.

In the present study, higher scores of anxiety and stress were associated with female and married students. This is in line with previous literature which suggests that female medical students have higher rates of symptoms of depression, anxiety, and stress $[37,38]$. However, our finding is inconsistent with result finding of Koochaki et al. [34] that noted no gender difference in level of stress among Iranian medical students. The possible explanation for this difference may be setup difference, technological difference, and curricular approach. Also, these studies have used different measuring tool.

In the present study, the prevalence of khat chewing was found to be $21.5 \%$. And, the level of stress was higher among khat chewers than nonchewer students. The possible reason is that khat stimulates adrenocortical function. This finding suggests that khat chewers show diversified psychological symptoms including stress, anxiety, depression, and emotional instability; this is due to its content of cathinone and cathine, which causes an increase in the release of cortisol, norepinephrine, and dopamine from presynaptic storage site (sympathomimetic effect) in the body of chewers. Consequently, the respondents experience psychostimulatory effects such as excitement and talkativeness initially. Then, they develop excessive worry, depressed mood, and tension $[39,40]$. The other possible justification is the socioeconomic problems caused due to increased demand of money to buy khat.

In the present study, the prevalence of cigarette smoking among medical students was $15.4 \%$. As reported by Parrott [41], smokers often mistakenly report that cigarettes help relieve feelings of stress, but the fact is that it does not alleviate stress. However, the stress levels of adult smokers are slightly higher than those of nonsmokers [5]. The level of stress was increased as they develop regular patterns of smoking and cessation of smoking reduces stress.
Furthermore, chronic nicotine intake affects the brain reward system function which results in depression and anxiety symptoms [42].

In the present study, the prevalence of alcohol consumption among medical students was $33.8 \%$, which is consistent with studies reported by Kalayu et al. [43]. However, it was a bit higher than study reported by Seipone et al. [44], because studies have used different measuring tool. In the present study, alcohol drinking predisposes students to mental illness. Alcohol largely affects the brain and the endocrine system. Alcohol changes levels of serotonin and other neurotransmitters in the brain. This can make stress and stress related-illness worse. Furthermore, alcohol actually increases the stress response by stimulating production of stress hormones. Alcohol directly activates the hypothalamus-pituitary-adrenal axis to produce excess cortisol [5].

\section{Conclusion}

Healthy medical students are likely to become healthy doctors. To sum up, overall prevalence rate of depression, anxiety, and stress is alarmingly high among Arsi University medical students. Monthly income, residency, and alcohol drinking were identified as risk factors of both depression and stress. In addition to other mentioned factors, educational year and khat chewing were identified as risk factors of stress. However, gender, marital status, educational year, residency, and cigarette smoking were identified as risk factors of anxiety. Besides, stress reduction interventions and implementation of structured orientation program that addresses issues like expectations for each phase, how students are going to be evaluated, how to cope, and how to get through each phase smoothly were recommended. In addition to awareness creation about the adverse effect of substance use, establishing student counselling center in the campus with qualified staff is also highly recommended. Family or close friend problem (recent death or accident), distance from family, frequency of money sent, and being first from home to go far were not assessed.

\section{Data Availability}

The datasets used and/or analyzed during the current study are available from the corresponding author on reasonable request.

\section{Conflicts of Interest}

The authors declare that there are no conflicts of interest regarding the publication of this paper.

\section{Acknowledgments}

The authors are grateful to College of Health Sciences Research and Community Office of Arsi University, all medical students of Arsi University, and data collectors as well as supervisors for their support and contribution. 


\section{References}

[1] G. Mythri, M. Manjunath, and B. Girish, "A study on perceived stress and sources of stress among first year medical students in Shivamogga," International Journal of Neurology, vol. 1, pp. 19-26, 2016.

[2] M. Marcus, M. Yasamy, M. van Ommeren, D. Chisholm, and S. Saxena, Depression: A Global Public Health Concern, pp. 6-8, WHO, Department of Mental Health and Substance Abuse., New York, NY, USA, 2012.

[3] E. A. Guthrie, D. Black, C. M. Shaw, J. Hamilton, F. H. Creed, and B. Tomenson, "Embarking upon a medical career: psychological morbidity in first year medical students," Medical Education, vol. 29, no. 5, pp. 337-341, 1995.

[4] M. S. Sherina, L. Rampal, and N. Kaneson, "Psychological stress among undergraduate medical students," The Medical Journal of Malaysia, vol. 59, pp. 207-211, 2004.

[5] L. Melaku, A. Mossie, and A. Negash, "Stress among medical students and its association with substance use and academic performance," Journal of Biomedical Education, vol. 2015, pp. 1-9, 2015.

[6] S. Mancevska, L. Bozinovska, J. Tecce, J. Pluncevik-Gligoroska, and E. Sivevska-Smilevska, "Depression, anxiety and substance use in medical students in the Republic of Macedonia," Bratislavske Lekarske Listy, vol. 109, pp. 568-572, 2008.

[7] Z. Mehanna and S. Richa, "Prévalence des troubles anxiodépressifs chez les étudiants en médecine," L'Encéphale, vol. 32, no. 6, pp. 976-982, 2006.

[8] I. Singh and A. Jha, "Anxiety, optimism and academic achievement among students of private medical and engineering colleges: a comparative study," Journal of Educational and Developmental Psychology, vol. 3, pp. 222-233, 2013.

[9] D. Eisenberg, N. Speer, and J. B. Hunt, "Attitudes and beliefs about treatment among college students with untreated mental health problems," Psychiatric Services, vol. 63, no. 7, pp. 711-713, 2012.

[10] M. Dahlin, N. Joneborg, and B. Runeson, "Stress and depression among medical students: a cross-sectional study," Medical Education, vol. 39, no. 6, pp. 594-604, 2005.

[11] R. Vaz, E. Mbajiorgu, and S. Acuda, "A preliminary study of stress levels among first year medical students at the University of Zimbabwe," Central African Journal of Medicine, vol. 44, pp. 214-219, 1998.

[12] T. C. Adam and E. S. Epel, "Stress, eating and the reward system," Physiology \& Behavior, vol. 91, no. 4, pp. 449-458, 2007.

[13] M. Macht, "How emotions affect eating: a five-way model," Appetite, vol. 50, no. 1, pp. 1-11, 2008.

[14] A. M. Lemieux, M. Nakajima, R. Saif-Ali, M. Al-Habori, A. Dokam, and M. al'Absi, "Anger, anxiety, and depressive affect as predictors of stress-induced cortisol production in khat and tobacco users," Addictive Behaviors, vol. 82, pp. 195-201, 2018.

[15] E. Martín-Merino, A. Ruigómez, M.-A. Wallander, S. Johansson, and L. A. Garcia-Rodriguez, "Prevalence, incidence, morbidity and treatment patterns in a cohort of patients diagnosed with anxiety in UK primary care," Family Practice, vol. 27, no. 1, pp. 9-16, 2010.

[16] J. R. Crawford and J. D. Henry, "The depression anxiety stress scales (DASS): normative data and latent structure in a large non-clinical sample," British Journal of Clinical Psychology, vol. 42, no. 2, pp. 111-131, 2003.
[17] T. D. Tran, T. Tran, and J. Fisher, "Validation of the depression anxiety stress scales (DASS) 21 as a screening instrument for depression and anxiety in a rural communitybased cohort of northern Vietnamese women," BMC Psychiatry, vol. 13, no. 1, p. 24, 2013.

[18] Y. Yeshaw and A. Mossie, "Depression, anxiety, stress, and their associated factors among Jimma University staff, Jimma, Southwest Ethiopia, 2016: a cross-sectional study," Neuropsychiatric Disease and Treatment, vol. 13, pp. 2803-2812, 2017.

[19] R. C. B. Vignola and A. M. Tucci, "Adaptation and validation of the depression, anxiety and stress scale (DASS) to Brazilian Portuguese," Journal of Affective Disorders, vol. 155, pp. 104-109, 2014.

[20] H. A. d. Silva, M. Passos, M. H. P. d. Passos et al., "Short version of the depression anxiety stress scale-21: is it valid for Brazilian adolescents?" Einstein (São Paulo), vol. 14, no. 4, pp. 486-493, 2016

[21] B. O. James, I. F. Thomas, J. O. Omoaregba et al., "Psychosocial correlates of perceived stress among undergraduate medical students in Nigeria," International Journal of Medical Education, vol. 8, pp. 382-388, 2017.

[22] S. Basudan, N. Binanzan, and A. Alhassan, "Depression, anxiety and stress in dental students," International Journal of Medical Education, vol. 8, pp. 179-186, 2017.

[23] B. Kulsoom and N. A. Afsar, "Stress, anxiety, and depression among medical students in a multiethnic setting," Neuropsychiatric Disease and Treatment, vol. 11, pp. 1713-1722, 2015.

[24] S. Iqbal, S. Gupta, and E. Venkatarao, "Stress, anxiety and depression among medical undergraduate students and their socio-demographic correlates," The Indian Journal of Medical Research, vol. 141, pp. 354-357, 2015.

[25] M. Fuad, B. Al-Zurfi, M. Abdelqader, M. Abu Bakar, M. Elnajeh, and M. Abdullah, "Prevalence and risk factors of stress, anxiety and depression among medical students of a private medical university in Malaysia," Malaysian Journal of Public Health Medicine, vol. 16, pp. 87-92, 2016.

[26] I. L. D. Moutinho, N. Maddalena, N. d. C. P. Maddalena et al., "Depression, stress and anxiety in medical students: a crosssectional comparison between students from different semesters," Revista da Associação Médica Brasileira, vol. 63, no. 1, pp. 21-28, 2017.

[27] P. Mehta, K. Thekdi, M. Rokad, K. Patel, A. Bhadu, and P. Sorani, "Exploratory study to access anxiety, depression and stress among medical students, freshly starting their medical education in a medical college. Scholars," Journal of Applied Medical Sciences, vol. 1, pp. 819-822, 2013.

[28] S. Patil, S. Sadhanala, and S. BansodeGokhe, "Study of depression, anxiety and stress among undergraduate medical students of A teaching medical institution," National Journal of Community Medicine, vol. 9, pp. 566-569, 2018.

[29] A. Al-Ani Radeef and F. Ghazi, "Depression, anxiety and stress among undergraduate science students in Malaysia," in Proceedings of the 17th Johor Mental Health Conference, Malaysia, April 2015.

[30] F. Rab, R. Mamdou, and S. Nasir, "Rates of depression and anxiety among female medical students in Pakistan," Eastern Mediterranean Health Journal, vol. 14, pp. 126-133, 2008.

[31] S. Ajay and P. Vijay, "A study of stressors in medical college students (Hostilities) in Northern Maharashtra," Journal of Indian Academy of Forensic Medicine, vol. 35, pp. 227-229, 2013. 
[32] D. Kunwar, A. Risal, and S. Koirala, "Study of depression, anxiety and stress among the medical students in two medical colleges of Nepal," Kathmandu University Medical Journal, vol. 53, pp. 22-26, 2016.

[33] H. Liaqat, U. Choudry, A. Altaf et al., "Deranged mental homeostasis in medical students: evaluation of depression anxiety and stress among home and hostel students," Acta Psychopathol, vol. 3, p. 1, 2017.

[34] G. M. Koochaki, A. Charkazi, A. Hasanzadeh et al., "Prevalence of stress among Iranian medical students: a questionnaire survey," Eastern Mediterranean Health Journal, vol. 17, no. 7, pp. 593-598, 2011.

[35] M. A. Fredericks and P. Mundy, "The relationship between social class, stress-anxiety responses, professional attitudes, and National Board scores of students in a medical school," Journal of the National Medical Association, vol. 60, pp. 271-278, 1968.

[36] T. Alvi, F. Assad, M. Ramzan, and F. A. Khan, "Depression, anxiety and their associated factors among medical students," Journal of the College of Physicians and Surgeons-Pakistan: JCPSP, vol. 20, pp. 122-126, 2010.

[37] V. Hope and M. Henderson, "Medical student depression, anxiety and distress outside North America: a systematic review," Medical Education, vol. 48, no. 10, pp. 963-979, 2014.

[38] A. M. Bassols, L. S. Okabayashi, A. B. d. Silva et al., "First- and last-year medical students: is there a difference in the prevalence and intensity of anxiety and depressive symptoms?" Revista Brasileira de Psiquiatria, vol. 36, no. 3, pp. 233-240, 2014.

[39] M. Odenwald and C. Khatkonsum, "Chronic khat use and psychotic disorders: a review of the literature and future prospects," SUCHT, vol. 53, pp. 9-22, 2008.

[40] G. Cox and H. Rampes, "Adverse effects of khat: a review," Advances in Psychiatric Treatment, vol. 9, no. 6, pp. 456-463, 2003.

[41] A. C. Parrott, "Does cigarette smoking cause stress?" American Psychologist, vol. 54, no. 10, pp. 817-820, 1999.

[42] M. Souza and A. Markou, "Role of GABA and glutamate circuitry," Cold Spring Harbor Perspectives in Medicine, vol. 3, pp. 1-18, 2013.

[43] M. Kalayu, M. Andualem, and G. Yeshigeta, "Effect of substance use on academic achievement of health officer and medical students of Jimma University, Southwest Ethiopia," Ethiopian Journal of Health Sciences, vol. 19, pp. 155-163, 2009.

[44] B. Seipone, G. Caroline, and B. Shyngle, "Stress and alcohol use among college students: a case of Molepolole College students," IOSR Journal of Humanities and Social Science, vol. 8, pp. 1-6, 2013. 